\title{
IMPACT OF SYSTEM STRENGTH AND HVDC CONTROL STRATEGIES ON DISTANCE PROTECTION PERFORMANCE
}

\author{
Di Liu ${ }^{1}$, Qiteng Hong ${ }^{1}$, Adam Dyśko', Dimitrios Tzelepis ${ }^{1}$, Guangya Yang ${ }^{2}$, Campbell \\ Booth $^{1}$, Ian Cowan ${ }^{3}$, Bharath Ponnalagan ${ }^{3}$
}

\author{
${ }^{I}$ Department of Electronic and Electrical Engineering, University of Strathclyde, Glasgow, United Kingdom \\ ${ }^{2}$ Technical University of Denmark, Lyngby, Denmark \\ ${ }^{3}$ The National HVDC Centre, Glasgow, United Kingdom \\ *q.hong@strath.ac.uk
}

\begin{abstract}
Keywords: HARDWARE-IN-THE-LOOP TESTS, DISTANCE PROTECTION, HVDC SYSTEM, SYSTEM STRENGTH, SYNCHRONOUS CONDENSER.
\end{abstract}

\begin{abstract}
This paper presents comprehensive studies and tests for evaluating the impact of reduced system strength and different control strategies used by HVDC systems on the performance of distance protection. A Hardware-In-the-Loop (HIL) test setup is established to enable realistic testing of physical relays being used in the system, where simulated voltage and current waveforms are injected into the distance protection relay via an analogue amplifier, and the relay tripping signal is fed back to simulation and recorded for protection performance analysis. In the simulation, a reduced but representative transmission network model, which includes a Modular Multilevel Converter (MMC) based HVDC system, a synchronous condenser (SC), and a two-level converter representing non-synchronous generation (NSG), is developed in RSCAD for the RTDS simulator. The model can be flexibly configured to reflect different levels of system strength and synchronous compensation applied at the HVDC site. The HVDC system is implemented with a flexible controller, which can replicate typically used control strategies during faults (e.g. balanced current mode to eliminate negative sequence current, and constant active and reactive power modes to suppress the oscillations on the active and reactive power respectively), allowing the user to inject different levels of negative sequence current. From the studies, it was found that with decreased system strength, the impact of the HVDC system on the distance protection becomes apparent, i.e. protection performance could be compromised with delayed operation, and such impact, to some extent, is subject to the control strategies applied in the HVDC system. It was also observed that the installation of SC could facilitate the protection response, and such support is dependent on the SC capacity.
\end{abstract}

\section{Introduction}

To address the ambitious decarbonisation targets, power systems worldwide generally see a significant increase of converter-interfaced renewable generation and HVDC systems play a critical role in integrating and transmitting renewable energy [1]. In the GB system, there are presently 8 HVDC links with a total capacity of approximately $8 \mathrm{GVA}$ and the number is expected to increase to over 30 links of more than 30 GVA by 2028 [2]. The rapidly increasing penetration of the converters introduces significant challenges to the operation of power system, and one of the key challenges is the reliable operation of protection systems [3]. Unlike conventional synchronous generators, the fault currents contributed by converters are limited and the fault behaviour is mainly determined by the embedded controller [4]. As a result, with the significant increase of converters in the system, there is an increasing concern that the distance protection performance could be compromised. Therefore, it is crucial to conduct a full assessment of distance protection performance under a wide range of system operating conditions and future energy scenarios.

The potential issues on distance protection performance in a converter-dominated system have been investigated in various pieces of work. The research in [5] reveals that the distance protection could suffer from undesired tripping delays, loss of zone discrimination in the transmission network with high amounts of converters. In [6], the impact of Voltage Source Converter (VSC) based HVDC on distance protection is evaluated, where the VSC-HVDC employs the balanced current control strategy [7] to supresses the negative sequence current. From this paper, the distance relay was found to experience under-reach and fault type diagnosis issues. Similar research activities are reported in [8][9]. However, a key limitation of the aforementioned studies is that they only assume the HVDC system is implemented with a specific type of control strategy but do not consider the impact when different types of HVDC controllers are used. To fill this gap, the study in [10] employs a flexible controller [11] to control the power features of the VSC during faults, thus allowing the emulation of different typically used HVDC control strategies to be conducted and tested against their impact on distance protection performance. However, the work does not consider the impact when there are different levels of system strength and only covers scenarios with unbalanced faults.

This paper presents comprehensive studies and tests for evaluating the impact of varied HVDC control strategies, system strength and the level of synchronous compensation on the distance relay performance under both balanced and unbalanced faults. The flexible controller in [12] is used for the MMC-HVDC system to simulate the three typically used 


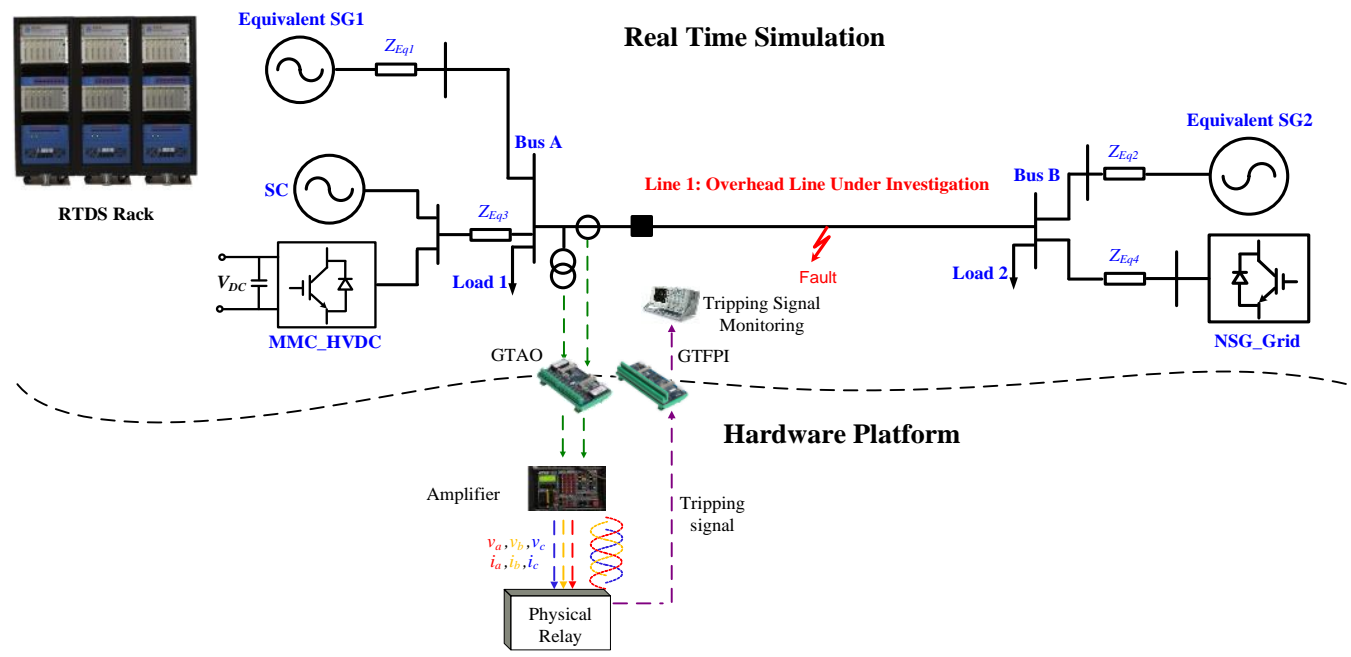

Fig. 1 Schematic of the developed RTDS network and HIL platform.

control strategies during faults, including the balanced current controller, constant active and reactive power controllers. The studies are conducted using a Hardware-In-the-Loop (HIL) test setup to enable realistic testing of actual physical relays being used in the system, where simulated voltage and current waveforms are injected to a commercially-available distance protection relay via an analogue amplifier and the relay tripping signal is fed back to the simulation and recorded for protection performance analysis.

The rest of the paper is organised as follows. In Section 2, an overview of the network model constructed in RTDS for testing distance protection performance and the HIL test platform is provided. Section 3 presents the test results for evaluating the protection performance under different system strengths and HVDC control strategies. Section 4 provides the conclusions of this paper.

\section{Real Time Hardware-in-the-Loop (HIL) Setup for Distance Protection Testing}

\subsection{Overview of the Hardware-In-the-Loop Setup}

For testing the distance protection performance under varied system strength and HVDC control strategies, a HIL test setup has been established as shown in Fig. 1. In the simulation part, a reduced but representative network model has been developed, which is capable of being configured to represent with different system strengths and contains controllers that could emulate typically used HVDC control strategies, which are further discussed in section 2.2-2.4. The simulation of the network model is run in real time and the simulated threephase instantaneous voltage and current waveforms at the relay location (i.e. Bus A) are stepped down to the secondary quantities via voltage and current transformer (VT and CT) models provided by RSCAD [13]. The stepped down voltage and current waveforms are further scaled so that they can be output through the analogue output card (i.e. GTAO card), which subsequently act as the inputs to the analogue amplifier. The amplifier will then amplify the voltage and current signals to the level that is equivalent to the actual secondary voltage and current in the real system, thus emulating the realistic operating conditions for the relay.

The output from the relay is the tripping signal, which is fed back to the simulation via a digital input card (i.e. GTFPI card as shown in Fig. 1) and recorded for monitoring the relay's tripping decision and the delay of the tripping action. The relay is configured with the Mho characteristic and two protective zones ('zone 1' and 'zone 2') are investigated. Zone 1 is configured to cover the $80 \%$ of the line length with instantaneous operation, while Zone 2 is set with an impedance equivalent to $120 \%$ of the line length and a delay of $500 \mathrm{~ms}$.

\subsection{Overview of the network model}

A schematic of the developed real time network model is shown in the Real Time Simulation part in Fig. 1. The model is developed in RSCAD software and simulated on the RTDS real time platform. In this model, the equivalent SG1 and SG2 represent the equivalent "lumped" synchronous generation sources at the two busbars $\mathrm{A}$ and B. $Z_{E q 1}$ and $Z_{E q 2}$ are the equivalent impedance of SG1 and SG2. MMC-HVDC, NSG_Grid and SC represent the MMC-HVDC system, nonsynchronous generation and synchronous condenser respectively. $Z_{E q 3}$ and $Z_{E q 4}$ are the equivalent impedances between HVDC system/the NSG unit and the buses. Load 1 and Load 2 are the equivalent loads connected at the two busbars. The investigated distance relay is installed at the Bus A and Line 1 is the protected line. By properly configuring the network model, an equivalent operating condition of a selected circuit in the power network can be recreated. Furthermore, the model can also be flexibly configured to emulate scenarios with the HVDC system of different capabilities and controllers, the varied penetration level of renewables and synchronous compensations, different system strength, etc.

\subsection{Modelling of the MMC-HVDC, NSG and SC}

To emulate the most typically used control strategies at the HVDC system, the dual-sequence current control [14] is implemented to control the positive and negative sequence components of the HVDC system with a flexible outer power controller designed based on (1) [12]. In (1), $\bar{P}$ and $\bar{Q}$ are the average real and reactive power, which are the power references given by the user. $v_{d}^{+}, v_{q}^{+}$and $v_{d}^{-}$and $v_{q}^{-}$are the positive sequence and negative sequence voltage and in $d$ and $q$ axes. The $i_{\text {dref }}^{+}, i_{\text {qref }}^{+}$and $i_{\text {dref }}^{-}, i_{\text {qref }}^{-}$are the positive and negative sequence current reference for the inner current controller. The variable $K_{\text {mode }}$ determines the type of the controller of the HVDC system. For example, when $K_{\text {mode }}$ equals 1, the HVDC system will supress the oscillation ripples 
on the active power; when $K_{\text {mode }}$ equals 0 , the HVDC system will mitigate the negative sequence current; when $K_{\text {mode }}$ equals -1 , the HVDC system will supress the ripples on the reactive power during faults. More detail on the various HVDC control strategies is reported in [12].

$$
\left[\begin{array}{l}
i_{\text {dref }}^{+} \\
i_{\text {qref }}^{+} \\
i_{\text {dref }}^{-} \\
i_{\text {qref }}^{-}
\end{array}\right]=\left[\begin{array}{cc}
\frac{+v_{d}^{+}}{D^{\prime}} & \frac{+v_{q}^{+}}{E^{\prime}} \\
\frac{+v_{q}^{+}}{D^{\prime}} & \frac{-v_{d}^{+}}{E^{\prime}} \\
\frac{-K_{\text {mode }} v_{d}^{-}}{D^{\prime}} & \frac{+K_{\text {mode }} v_{q}^{-}}{E^{\prime}} \\
\frac{-K_{\text {mode }} v_{q}^{-}}{D^{\prime}} & \frac{-K_{\text {mode }} v_{d}^{-}}{E^{\prime}}
\end{array}\right] \times\left[\begin{array}{c}
\bar{P} \\
\bar{Q}
\end{array}\right]
$$

Where the $D^{\prime}$ and $E^{\prime}$ are defined as:

$$
\begin{aligned}
& D^{\prime}=\left(\left(v_{d}^{+}\right)^{2}+\left(v_{q}^{+}\right)^{2}\right)-K_{\text {mode }}\left(\left(v_{d}^{-}\right)^{2}+\left(v_{q}^{-}\right)^{2}\right) \\
& E^{\prime}=\left(\left(v_{d}^{+}\right)^{2}+\left(v_{q}^{+}\right)^{2}\right)+K_{\text {mode }}\left(\left(v_{d}^{-}\right)^{2}+\left(v_{q}^{-}\right)^{2}\right)
\end{aligned}
$$

For the design of the converter interfaced NSG, the conventional PQ control strategy [15] is implemented. The controller of SC is provided by RSCAD [16], which is based on the IEEE type 1 excitation system [17].

\subsection{Configuration of the system strength}

The system strength is typically indicated by the short circuit ratio (SCR), which is defined as a ratio of the total three phase fault level to the rating of the HVDC system. However, from Fig. 1, it can be seen that the HVDC unit and the distance relay are connected to Bus A, and when faults occur on Line 1, the stability of the HVDC system is mainly determined by the local voltage of Bus A and the fault current seen by the relay is mainly contributed from the Bus A side. Therefore, for simplicity, in this study, the SCR has been re-defined as (2).

$$
S C R_{\text {Bus } A}=\frac{F L_{B u S A}}{S_{H V D C}}
$$

Where the $F L_{B u s}$ is the fault level contributed from the generation at Bus A side, i.e. the fault infeed from SG 1, which can be controlled by changing the values of $Z_{e q 1}$; and $S_{H V D C}$ is the capacity of the HVDC system. Therefore, through the configuration of the value of $Z_{e q 1}$, the system strength at Bus A can be adjusted.

\section{Case Studies}

\subsection{Overview of the studied cases}

In reality, there are a wide range of factors that could affect the performance of the distance protection relay. However, owing to the space limitation, the case studies designed in this section are mainly focused on investigating the impacts of different HVDC controllers and different levels of SCRs, and different levels of synchronous compensation on distance protection performance. The cases investigated are summarised in Table 1 and described as follows:

- Case 1: the impact of the varied types of the HVDC control modes, including constant active power control (CP) with $K_{\text {mode }}=1$, balanced current control (BC) with $K_{\text {mode }}=0$, and constant reactive power control (CQ) with $K_{\text {mode }}=-1$, under asymmetrical faults.

- Case 2: the impact of the system strength with $S C R_{B u s ~}=$ 2, 3 and 5 under asymmetrical faults.

- Case 3: the impact of SC capacity at the HVDC site under asymmetrical faults.
Table 1 The information about the case studies.

\begin{tabular}{ccccc}
\hline $\begin{array}{c}\text { Case } \\
\text { Number }\end{array}$ & $S C R_{\text {Bus A }}$ & $\begin{array}{c}K_{\text {mode }} \\
\text { values }\end{array}$ & $\begin{array}{c}\text { SC capacity } \\
\text { (MVar) }\end{array}$ & $\begin{array}{c}\text { Fault } \\
\text { Type }\end{array}$ \\
\hline 1 & 3 & $1,0,-1$ & N/A & AG,AB \\
2 & $2,3,5$ & 1 & N/A & AG,AB \\
3 & 2 & 1 & $0,150,300,500$ & AG,AB \\
4 & $7,5,2$ & 0 & N/A, 500, 1800 & ABCG \\
\hline
\end{tabular}

Table 2 Parameters of the studied network

\begin{tabular}{cc}
\hline Parameters & Values \\
\hline$V_{S}$ & $400 \mathrm{kV}$ \\
$Z_{\text {Line }}$ & $1.15+\mathrm{j} 12.65 \Omega$ \\
$Z_{E q 1}$ & $4.84+\mathrm{j} 53.12 \Omega$ \\
$Z_{E q 2}$ & $4.84+\mathrm{j} 53.12 \Omega$ \\
$Z_{E q 3}$ & $0.29+\mathrm{j} 3.12 \Omega$ \\
$Z_{E q 4}$ & $0.29+\mathrm{j} 3.12 \Omega$ \\
$P_{H V D C}, Q_{H V D C}$ & $1000 \mathrm{MW} ; 0 \mathrm{MVar}$ \\
$P_{N S G}, Q_{N S G}$ & $2000 \mathrm{MW} ; 1000 \mathrm{MVar}$ \\
$P_{L 1}, Q_{L 1}$ & $100 \mathrm{MW} ; 50 \mathrm{MVar}$ \\
$P_{L 2}, Q_{L 2}$ & $2000 \mathrm{MW} ; 900 \mathrm{MVar}$ \\
\hline
\end{tabular}

- Case 4: the impact of system strength and synchronous compensation under symmetrical faults.

The parameters of the network model are presented in Table 2, where the shown impedance values are in positive sequence. The value of $Z_{E q 1}$ corresponds to the case with $S C R_{B u s A}=3$, which could be adjusted when investigating the impact of varied system strength. The maximum tolerable currents from the HVDC system and NSG are restricted to 1.2 p.u.

\subsection{HIL test results from case studies}

\subsubsection{Case 1: Impact of different HVDC control strategies}

In this case study, a Phase A to Ground fault (AG) and a Phase A to $\mathrm{B}$ fault $(\mathrm{AB})$ with $1 \Omega$ resistance are simulated at locations with a distance of $60 \%$ to $85 \%$ of the line length to the relay location at a $5 \%$ step. The response time of the relay, which is defined as the time between the fault inception and the relay tripping signal recorded in RTDS, is shown in Fig. 2 (a). For the AG faults, the distance relay has the similar response for the HVDC system using balanced current and constant reactive power control strategies. However, a severe delay (almost $400 \mathrm{~ms}$ ) occurs for the fault at $75 \%$ of the line when the HVDC system employs the constant active power controller, which is much longer than the required protection operating time of $140 \mathrm{~ms}$ as specified in [18]. In the case of $\mathrm{AB}$ faults, the relay trips in Zone 2 for the fault at Zone $1(75 \%)$ when the constant active power and balanced current controllers are implemented. Based on these scenarios, it can be concluded that the choice of the HVDC controller has clear influence on the distance protection under the same system condition, and the control strategies of $\mathrm{CP}$ and $\mathrm{BC}$ appear to suffer from the tripping delay and under-reach issue for the faults close to the zone's boundary.

\subsubsection{Case 2: Impact of system strength}

By changing the values of $S C R_{B u s ~}$, the impact of the system strength is evaluated in this section. According to Case 1, the distance relay appears to potentially suffer from the time delay and under-reach issues when HVDC system operates under the constant active power control strategy, therefore, this strategy is chosen as the example for the test in this section. The same 

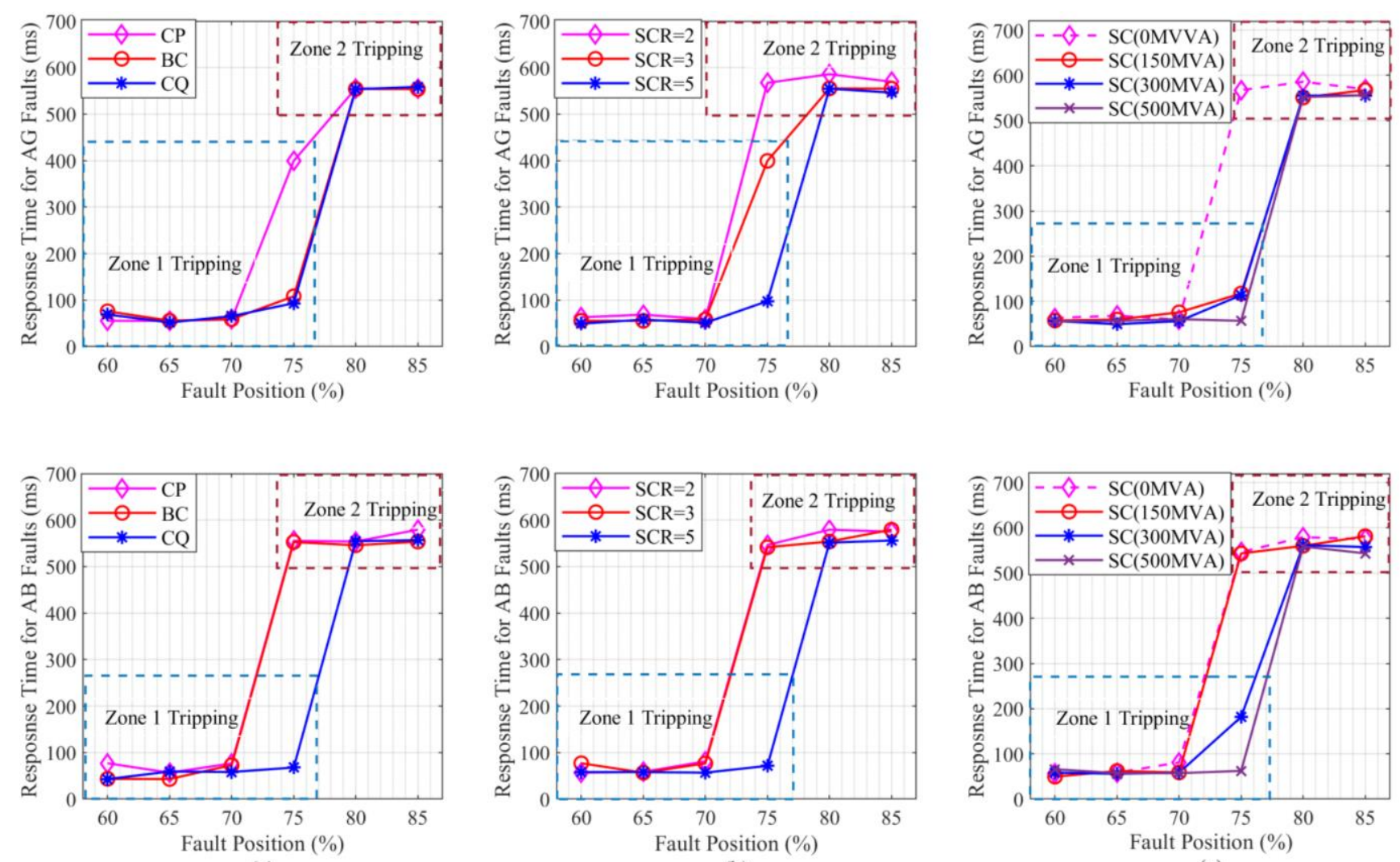

(a)

(b)

(c)

Fig. 2. Tripping time of physical distance relay for, (a) different control strategies of HVDC system, (b) network with varied system strength, (c) different levels of the synchronous compensation.
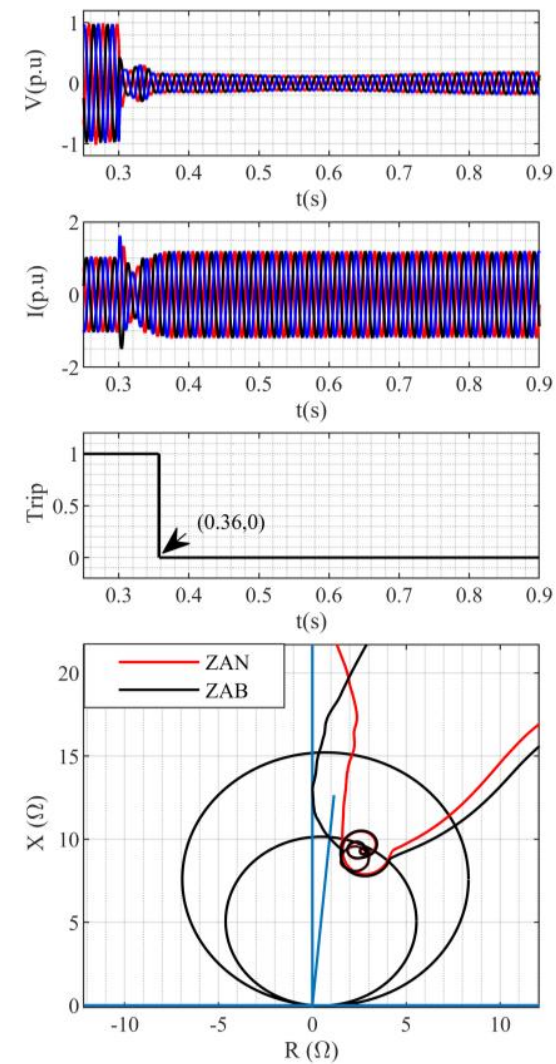

(a)
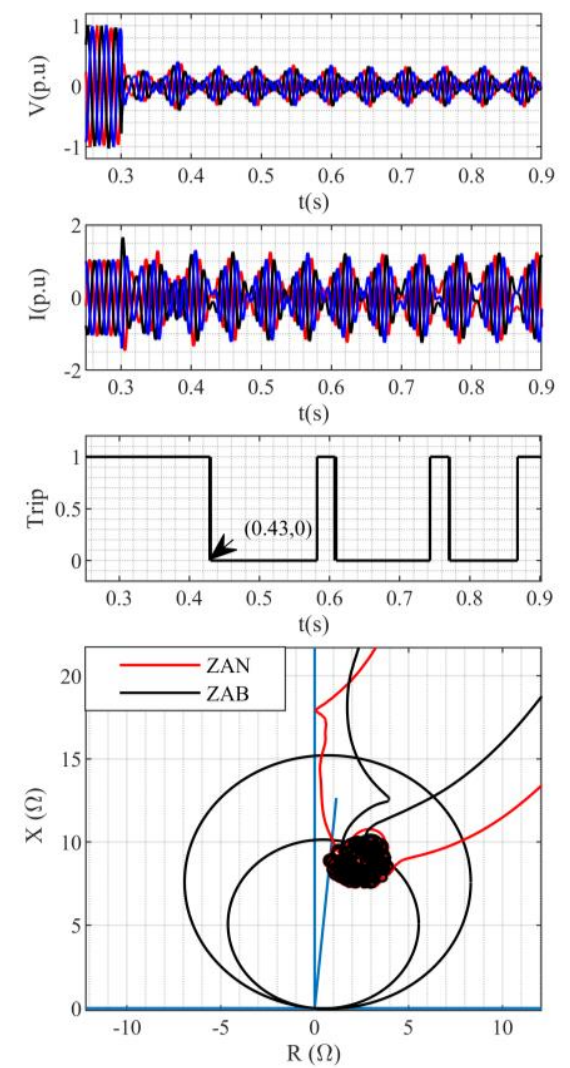

(b)
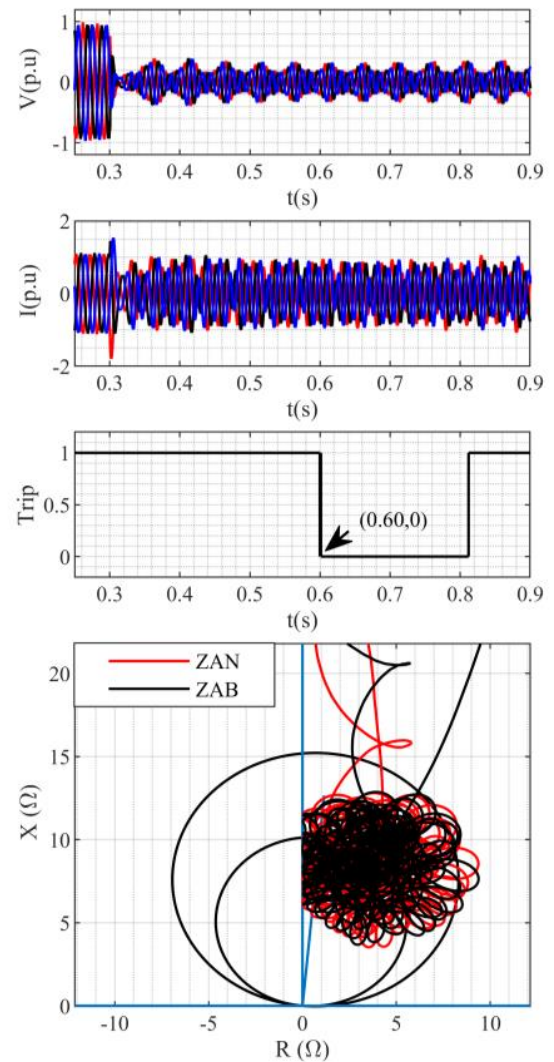

(c)

Fig. 3 The behaviours of HVDC system and distance relay under ABCG fault at $70 \%$ in the case of, (a) $S C R_{B u s}=7$ without compensation, (b) $S C R_{B u s A}=5$ without compensation, (c) $S C R_{B u s A}=2$ without compensation. 
faults in Case 1 are simulated on the protected line. Based on the results in Fig. 2 (b), the distance relay trips correctly within the required time when the system is relatively strong (i.e. $\left.S C R_{B u s A}=5\right)$. In the case where the network $S C R_{B u s A}=3$, the relay has a $400 \mathrm{~ms}$ delay for the AG fault at $75 \%$ of the line and it trips falsely in zone 2 for $\mathrm{AB}$ fault at the same position. Furthermore, as the value of the $S C R_{B u s}$ decreases to 2 , the relay fails to effectively discriminate the correct fault zone in both types of the faults. The test results clearly suggest that the distance protection performance is more likely to be compromised with undesired delays and potentially undesirable tripping decision when the system strength decreases.

\subsubsection{Case 3: Impact of the synchronous condenser}

This case investigates the impact of integrating different capacities of SC at the HVDC site on distance protection. Based on the test results presented in previous sections, the distance relay performance tends to experience issues when the network has low system strength and HVDC system operates in the constant active power control mode. To highlight the impact of the synchronous condenser, the worstcase scenario, where $S C R_{\text {Bus A }}=2$ with the constant active power controller implemented in the HVDC system, is used as the reference case. The result of the reference case is shown by the dashed line in Fig. 2 (c). It can be seen that the connection of the SC can mitigate the zone discrimination issue of the distance relay under AG faults and speeds up the tripping action, and such effect becomes more apparent as the capacity of the SC increases. Similar results can be observed for the AB faults, but the results of $\mathrm{AB}$ faults also reveal that the $\mathrm{SC}$ with small capacity may not be enough to correct the under-reach (when <150 MVA) and relay delayed operation issues (when $<300$ MVA) of the distance relay. The interpretation of the result is that by installing an SC at the HVDC site, the system strength at Bus A is effectively increased and the level of system strength increase is proportional to the SC capacity. This also aligns with the observation in Case 2 when the system strength is relatively high, the distance protection appears to have a low risk of experiencing compromised performance.

\subsubsection{Case 4: Impact of the system strength and synchronous} compensation under ABCG faults.

Unlike the previous three cases, in the symmetrical faults, there will be no presence of negative sequence voltage. The values of $v_{d}^{-}$and $v_{q}^{-}$in (1) will become zero, thus no matter what value $K_{\text {mode }}$ is used, the output current will always be three phase balanced. Additionally, in the case of the symmetrical faults with low impedance, the three-phase voltage at the HVDC terminal could drop to a very low level, this will cause the instability of the converter owing to the synchronisation issue of the Phase Locked Loop (PLL) [19]. In this case, the performance of the distance relay will be also affected. In this section, the distance protection is investigated in the network with varied values of the $S C R_{B u s ~}$ and the different levels of the synchronous compensation. The faults in this study are simulated at $70 \%$ of the protected line and the fault resistance is $1 \Omega$.

The simulation results without the SC are shown in Fig. 3, where the network with varied system strengths are discussed including values of the $S C R_{B u s}$ equal 7, 5 and 2 respectively. The voltage and current are the terminal three-phase voltages and currents measured from the RTDS. The trip signal is recorded from the physical relay. Moreover, to further figure out the relay behaviour, a distance relay model [20] with six impedance measuring blocks, named AG, BG, CG, AB, AC, $\mathrm{BC}$, is developed in Simulink to visualise the impedance locus during the faults to facilitate better understanding of the results It should be noted that the use of Simulink distance relay model to plot impedance locus is not the only option, and other similar protection models or impedance plotting tools could also be used. For the ABCG fault, all measuring blocks can sense the faults, therefore, the impedances measured by the $\mathrm{AG}$ and $\mathrm{AB}$ blocks are used as examples. When fault occurs in a relatively strong network $\left(S C R_{B u s ~}=7\right)$, based on the result of Fig. 3 (a), the HVDC system can maintain stable and the relay can trip in the correct zone. However, when the value of the $S C R_{B \text { us A }}$ decreases to 5, although the distance relay trips initially in zone 1, as seen Fig. 3 (b), the tripping signal shows some oscillation in the next several cycles and the impedance locus appears to have more severe swings. The worst scenario can be found when the $S C R_{\text {Bus } A}$ drops to 2 as shown in Fig. 3 (c), where the distance relay suffers from a severe delay (300 $\mathrm{ms}$ ) and the impedance locus becomes unstable.

It should be noted that, in this case study, the HVDC system starts to experience stability issues during ABCG faults with $S C R_{B u s A}=5$. This is not typically expected behaviour for such a system strength level. It is anticipated that this could be caused by the fact that the HVDC system has not been equipped with fault ride through capability and it does not inject a required amount of reactive current during faults as specified by the associated grid codes. These fault ride through issues will be considered in the next stage of the research.
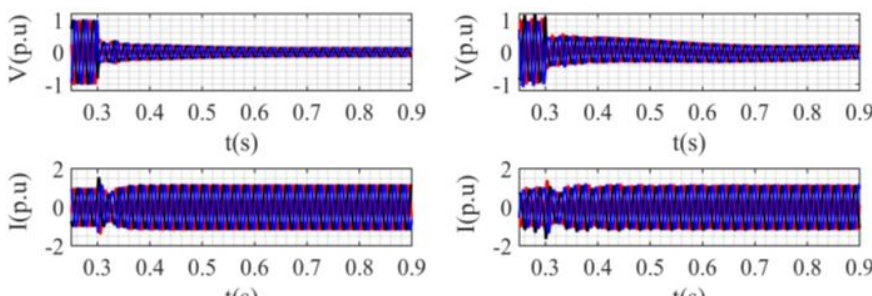

$\mathrm{t}(\mathrm{s})$

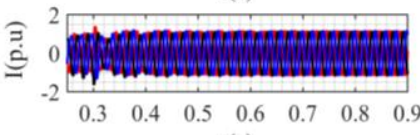

$\mathrm{t}(\mathrm{s})$

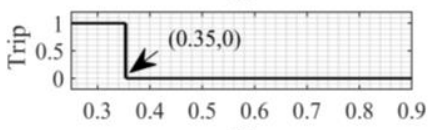

$\mathrm{t}(\mathrm{s})$
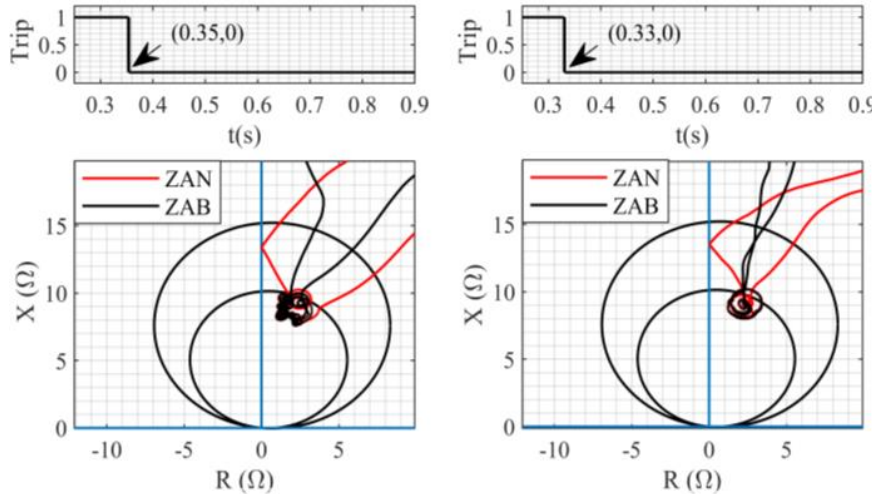
$\mathrm{t}(\mathrm{s})$

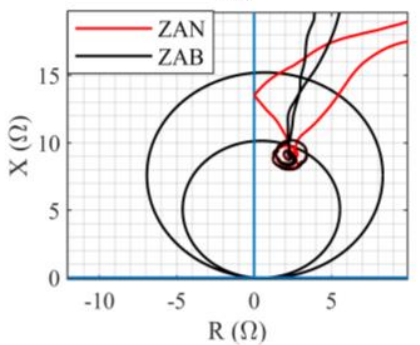

(b)

Fig. 4 The behaviours of HVDC system and distance relay under ABCG fault in the case of, (a) $S C R_{\text {Bus } A}=5$ with 500 MVA compensation, (b) $S C R_{\text {Bus } A}=2$ with 1800 MVA compensation.

As reported in [21], the connection of SC is beneficial to the distance protection performance. In this case, the SCs with the 
500 MVA and 1800 MVA capacities are connected to improve the performance of the distance relay and to maintain the stability of the HVDC system in the network with $S C R_{B \text { us } A}$ of 5 and 2 respectively. The capacities of SCs are the minimum capacities capable of recovering the distance protection operation and HVDC system stability. The results are shown in Fig. 4, and it can be seen that the performance of the distance relay and HVDC system appear to have clear improvement in terms of the protection operation time and the stability of the HVDC system with the assistance of the SCs. A higher level of synchronous compensation will be required for the network with the lower SCR.

\section{Conclusions}

In this paper, the impact of system strength, different HVDC control strategies and the level of synchronous compensation at the HVDC site on the performance of distance protection has been tested and analysed. The studies have been conducted using an HIL setup that allows injection of faults in a range of system conditions and HVDC control modes to a physical relay. It was found that, for asymmetrical faults, the distance relay performance does depend on the control strategy adopted by the HVDC system, especially when system strength is low. Specifically, for the faults near the boundary of the protection zone, under-reach issues and longer tripping delays are observed when the balanced current controller and constant active power controller are implemented to the HVDC system, while the constant reactive power control strategy appears to be more protection-friendly. For symmetrical faults, the HVDC controller strategy appears to have negligible effect due to the absence of negative sequence current. It was found that when the system becomes weak, the HVDC system could have the stability issues during the symmetrical faults, which could also cause comprised performance of distance protection.

It was also found that for all types of faults and HVDC control strategies, issues with compromised relay performance would only become apparent when the system strength is low. Therefore, the connection of the SC can help to mitigate these issues by enhancing the system strength. However, in some situations, a high level of synchronous compensation could be required if purely relying on the SC to address the protection issues, so the economy aspect should be further evaluated, along with other benefits that SCs can offer.

\section{Acknowledgements}

The work is sponsored by the National HVDC Centre and the Phoenix project led by SP Energy Networks in the UK.

\section{References}

[1] V. Telukunta, J. Pradhan, et al., "Protection challenges under bulk penetration of renewable energy resources in power systems: A review," in CSEE Journal of Power and Energy Systems, vol. 3, no. 4, pp. 365-379, Dec. 2017.

[2] National Grid ESO, "Interconnector Register by 30 July 2020," 2020.

[3] B. Kroposki et al., "Achieving a 100\% Renewable Grid: Operating Electric Power Systems with Extremely High Levels of Variable Renewable Energy," in IEEE Power and Energy Magazine, vol. 15, no. 2, pp. 61-73, March-April 2017.

[4] D. Liu, D. Tzelepis, A. Dyśko, and C. Booth, A novel protection scheme for inverter-dominated microgrid. Paper presented at
The $15^{\text {th }}$ International Conference on Developments in Power System Protection, Liverpool, UK.

[5] R. Li et al., "Impact of low (zero) carbon power systems on power system protection: a new evaluation approach based on a flexible modelling and hardware testing platform," in IET Renewable Power Generation, vol. 14, no. 5, pp. 906-913, 2020.

[6] M. Alam, H. Leite, N. Silva, and A. Carvalho, "Performance evaluation of distance protection of transmission lines connected with VSC-HVdc system using closed-loop test in RTDS," Electric Power Syst. Res., vol. 152, pp. 168-183, Nov. 2017.

[7] M. Rahman, D. Guo, G. Adam, A. Emhemed, and L. Xu, Development and Validation of Offline and Real-Time UserDefined Models of Alternative MMC Configurations, Strathclyde-UK National HVDC Centre collaborative research project, April 2018.

[8] C. Teixeira and H. Leite, "The influence of a VSC based HVDC link on distance protection relay assessed by CAPE software," 2017 IEEE Manchester PowerTech, Manchester, 2017, pp. 1-4.

[9] S. Tale, M. Mohan and K. P. Vittal, "Performance analysis of distance relay in an AC grid with VSC-HVDC connection," 2017 International Conference on Intelligent Computing, Instrumentation and Control Technologies (ICICICT), Kannur, 2017, pp. 1363-1368.

[10] J. Jia, G. Yang, A. H. Nielsen and P. Rønne-Hansen, "Impact of VSC Control Strategies and Incorporation of Synchronous Condensers on Distance Protection Under Unbalanced Faults," in IEEE Transactions on Industrial Electronics, vol. 66, no. 2, pp. 1108-1118, Feb. 2019.

[11] J. Jia, G. Yang and A. H. Nielsen, "A Review on Grid-Connected Converter Control for Short-Circuit Power Provision Under Grid Unbalanced Faults," in IEEE Transactions on Power Delivery, vol. 33, no. 2, pp. 649-661, April 2018.

[12] R. Kabiri, D. Holmes and B. McGrath, "Control of Active and Reactive Power Ripple to Mitigate Unbalanced Grid Voltages," in IEEE Transactions on Industry Applications, vol. 52, no. 2, pp. 1660-1668, March-April 2016.

[13] RTDS Technology Inc., "Real Time Digital Simulator Power System Components Manual".

[14] J. Jia, G. Yang, and N. Nielsen, Fault Analysis Method Considering Dual-Sequence Current Control of VSCs under Unbalanced Faults. Energies 2018, 11, 1660.

[15] J. Rocabert, A. Luna, F. Blaabjerg and P. Rodríguez, "Control of Power Converters in AC Microgrids," in IEEE Transactions on Power Electronics, vol. 27, no. 11, pp. 4734-4749, Nov. 2012.

[16] RTDS Technology Inc., "Real Time Digital Simulator Control Library Manual".

[17] IEEE Recommended Practice for Excitation System Models for Power System Stability Studies," in IEEE Std 421.5-2016 (Revision of IEEE Std 421.5-2005), pp.1-207, 26 Aug. 2016.

[18] The Grid Code, Issue 5 (2017, March.). National Grid plc, U.K.

[19] Ö. Göksu, R. Teodorescu, C. L. Bak, F. Iov and P. C. Kjær, "Instability of Wind Turbine Converters During Current Injection to Low Voltage Grid Faults and PLL Frequency Based Stability Solution," in IEEE Transactions on Power Systems, vol. 29, no. 4, pp. 1683-1691, July 2014.

[20] Network Protection \& Automation Guide, 2011.

[21] D. Tzelepis, E. Tsotsopoulou, V. Nikolaidis, A. Dyśko, V. Papaspiliotopoulos, Q. Hong, and C. Booth, Impact of synchronous condensers on transmission line protection in scenarios with high penetration of renewable energy sources. Paper presented at IET 15th International Conference on Developments in Power System Protection, Liverpool, UK. 